\title{
Imaging of enthesitis by an LED-based photoacoustic system
}

\author{
Janggun Jo $\odot,{ }^{a, \dagger}$ Guan Xu, ${ }^{\text {a,b, }, \dagger}$ Elena Schiopu, ${ }^{\mathrm{c}, *}$ David Chamberland, \\ Girish Gandikota, ${ }^{\mathrm{d}, *}$ and Xueding Wang ${ }^{\mathrm{a}, \mathrm{d}, *}$ \\ ${ }^{a}$ University of Michigan, Department of Biomedical Engineering, Ann Arbor, \\ Michigan, United States \\ ${ }^{b}$ University of Michigan, Department of Ophthalmology and Visual Sciences, \\ Ann Arbor, Michigan, United States \\ ${ }^{c}$ University of Michigan Medical School, Division of Rheumatology, \\ Department of Internal Medicine, Ann Arbor, Michigan, United States \\ ${ }^{\mathrm{d}}$ University of Michigan Medical School, Department of Radiology, Ann Arbor, \\ Michigan, United States
}

\begin{abstract}
Significance: One key pathological characteristic of seronegative spondyloarthropathy (SpA) is inflammation at the insertion of tendons and ligaments into the bone (enthesitis).

Aim: We explore the potential of the emerging photoacoustic (PA) imaging in diagnosis of SpA and review its feasibility in detecting SpA-associated Achilles tendon enthesitis.

Approach: A light-emitting diode (LED)-based PA and ultrasound combined system was employed. The PA images, both along the long and the short axes of each Achilles tendon insertion region, were acquired at 850-nm wavelength, which is sensitive in depicting increased blood volume (i.e., hyperemia). To assess the hyperemia indicating enthesis inflammation, two parameters were quantified in the imaged tendons, including the average intensity and the density of the color pixels in the pseudo-color PA images. Ten SpA patients, all of which met Assessment of SpA International Society (ASAS) criteria for SpA and were found to have Achilles enthesitis by clinical exam according to a board-certified rheumatologist, were included in the study.
\end{abstract}

Results: The PA and Doppler ultrasound imaging of Achilles enthesitis resulting from these $10 \mathrm{SpA}$ patients were compared to those from 10 healthy volunteers, leading to statistically significant differences $(p<0.05)$ in the applied $t$-tests.

Conclusions: This preliminary clinical study suggests that the LED-based PA imaging holds a promise for sensitive and objective assessment of SpA enthesitis in an outpatient setting of the rheumatology clinic.

(C) The Authors. Published by SPIE under a Creative Commons Attribution 4.0 Unported License. Distribution or reproduction of this work in whole or in part requires full attribution of the original publication, including its DOI. [DOI: 10.1117/1.JBO.25.12.126005]

Keywords: photoacoustic imaging; hyperemia; light-emitting diode; psoriatic arthritis; tendon inflammation.

Paper 200274RR received Sep. 2, 2020; accepted for publication Dec. 1, 2020; published online Dec. 17, 2020; corrected May 7, 2021.

\section{Introduction}

Seronegative spondyloarthropathies (SpA) [psoriatic arthritis (PsA), Ankylosing spondylitis (AS), reactive arthritis (ReA), arthritis associated with inflammatory bowel disease (IBD), and undifferentiated spondyloarthritis (uSpA)] are a heterogeneous family of inflammatory arthritides characterized by Assessment of SpondyloArthritis international Society (ASAS) as having

\footnotetext{
*Address all correspondence to Elena Schiopu, eschiopu@med.umich.edu; Girish Gandikota, ggirish@med.umich.edu; Xueding Wang, xdwang@umich.edu

${ }^{\dagger}$ Equal contribution
} 
inflammatory back pain, presence of enthesitis, dactylitis, and a variety of other extraarticular manifestations. PsA affects about $30 \%$ of patients with psoriasis in the United States. ${ }^{1-3}$ Early SpA treatment is challenged by reduced imaging sensitivity at early stages of pathology conditions, where symptoms are nonspecific and emerging after radiographic progression occurs, and the average time from onset of symptoms to diagnosis can be as long as 10 years. ${ }^{4}$ The lack of early diagnosis of SpA is due to limited pathognomonic clinical technology in specific imaging of the biomarkers of the disease. Although initially considered as a variant of rheumatoid arthritis (RA), SpA was later defined as a distinct clinical entity. ${ }^{3,5}$ Clinical assessment of physiological changes associated with $\mathrm{SpA}$ is also a problem. Unlike RA-related synovitis, enthesitis is more challenging to detect with ultrasound (US) imaging. Magnetic resonance imaging (MRI) remains an excellent choice for imaging enthesitis, but is costly and not easily accessible. Emerging clinical research on SpA has been focused on studies that identify or validate biomarkers that are sensitive to early diagnosis and disease progression and early responses to therapy.

The physiological symptoms such as hyperemia and hypoxia in soft tissue are directly associated with the pathological condition of the joints, tendons, and entheses affected by inflammatory arthritis. By monitoring these features using the emerging photoacoustic (PA) imaging technology, joint inflammation has been detected and evaluated in previous studies. ${ }^{6-13}$ Based on highly sensitive optical contrast, PA imaging offers excellent sensitivity in assessing hyperemia, ${ }^{11,12,14}$ which is an essential step in the inflammatory cascade and a result of chronic inflammation causing hypervascularization associated with an increased capillary network and distended veins. In addition, by performing dual-wavelength PA imaging, the hypoxia in arthritic joints as another important physiological symptom can be quantitatively measured. ${ }^{11}$ These previous studies on inflammatory joints have suggested that PA imaging, as an add-on to clinical US imaging, could offer additional and valuable diagnostic information for an earlier and more comprehensive assessment of inflammatory arthritis and responses to therapy.

Early symptoms of SpA are nonspecific and could be followed by a period of undifferentiated inflammatory arthritis that is difficult to distinguish from RA. Several distinct clinical features, such as hyperemia in tendons and entheses, distinguish SpA from other forms of inflammatory arthritis. In this initial clinical study on SpA patients, we, for the first time to the best of our knowledge, explored the feasibility of PA imaging in detecting inflammation in human Achilles entheses in patients who met the criteria for ASAS-defined SpA. In this study, a commercially available light-emitting diode (LED)-based PA imaging system was employed. A good performance of this system in detecting inflammation in arthritic joints has been reported. ${ }^{12,13}$ Using low-cost, small-size, portable, and clinically safe light sources such as LEDs as the illumination source, PA imaging has many advantages. It may accelerate the clinical translation of this emerging medical imaging technology by providing a safe, sensitive, easily adaptable, and clinically useful tool aiding early SpA diagnosis. Imaging results from the SpA patients were compared to those from the healthy volunteers, and the presence of inflammation was confirmed by PA and the routine musculoskeletal US Doppler imaging.

\section{Methods}

The LED PA imaging system used in this study was built by Cyberdyne (AcousticX, Cyberdyne, Tokyo, Japan), as shown in Fig. 1. The details of this system, including the safety for applications on human subjects, have been introduced in our previous publications. ${ }^{12,13,15,16}$ The system has two LED array bars located on both sides of a US transducer probe with an angle of 45 deg to the center plane of the probe (Fig. 1). The light source has a repetition rate of $4 \mathrm{kHz}$ and a pulse duration of $35 \mathrm{~ns}$. Each pulse provides light energy of $400 \mu \mathrm{J}$ at an 850-nm wavelength. The PA signals were acquired at each LED pulse. After processing the PA signals using a frequency bandpass filter, log compression, and time gain compensation, the PA images were reconstructed using the delay-and-sum method and then displayed in pseudocolor. The reconstructed PA image in pseudocolor was processed by the integrated software in AcousticX and displayed on the background gray scale US image after applying dynamic range control to enhance high intensity signals. A consistent gain of 56 was employed during PA imaging of all human tendons in this 
(a)

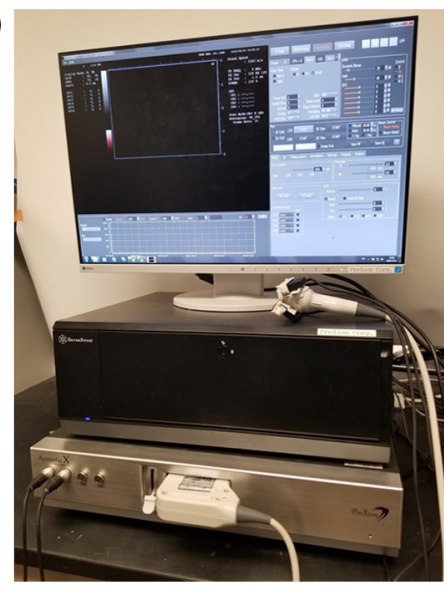

(b)

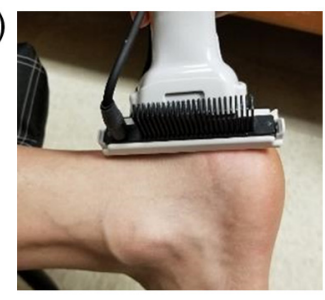

(c)

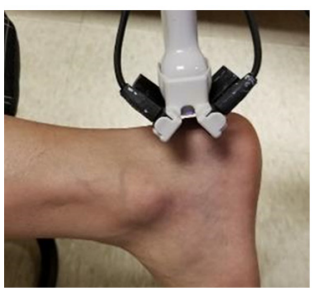

Fig. 1 LED-based PA imaging of tendon inflammation. (a) Photograph of the imaging system. (b) Patient's Achilles tendon scanned with the imaging probe along the long axis of the tendon.

(c) Patient's Achilles tendon scanned with the imaging probe along the short axis of the tendon.

study. Using the same level of gain, we can compare the PA image intensities between the patient and control groups. To enhance the signal-to-noise ratio, the PA images were averaged over 384 light pulses, leading to an imaging frame rate of $\sim 10 \mathrm{~Hz}$. The US images acquired using the same probe were displayed in a gray scale, also in real-time. When working with the 7-MHz linear probe with 128 elements, as employed in this study, this system offers a spatial resolution of $310-\mu \mathrm{m}$ lateral and $250-\mu \mathrm{m}$ axial, and an image depth up to $30 \mathrm{~mm}$ for PA imaging. ${ }^{12,13}$

In this study, conventional US Doppler imaging was used as the gold standard for confirming the hyperemia in the affected enthuses. ${ }^{17,18}$ Because the AcousticX imaging system does not provide the Doppler mode, the US Doppler images were acquired by a commercial US unit (Z.ONE PRO, Mindray, Mahwah, New Jersey) working with a linear probe (L14-5W, Mindray). The US Doppler images were acquired at a pulse repetition frequency of $1500 \mathrm{~Hz}$ and a scale of +7.5 to $-7.5 \mathrm{~cm} / \mathrm{s}$ to show hyperemia in the enthesis area both along and across the enthesis orientation. The same approximate imaging planes were revisited by the LED-based PA imaging system. The patients' entheses that did not show prominent flow in the US Doppler imaging were also scanned by the LED PA imaging in search of increased vascularity. The total scanning time, including both Doppler US and LED PA imaging of each human subject, was less than 10 min.

All procedures for human subjects in this study were approved by the Institutional Review Board (IRB) of the University of Michigan Medical School. We performed the study in accordance with the approved protocol. Ten SpA patients, who are either men or women and are over 18 years old, were recruited through the University of Michigan Rheumatology clinics. Boardcertified rheumatologists at the University of Michigan Medical School confirmed both the pathological condition of SpA, according to ASAS, and enthesitis. The normal control group includes 10 healthy volunteers who did not have symptoms or signs of enthesitis and no clinical record of any inflammatory arthritis. All participants provided written informed consent.

\section{Results}

Figure 2 shows the representative imaging results from an SpA patient. In gray-scale B-mode US images, the areas of distal Achilles tendon that connect the heel bone (Calcaneum) with the calf muscles can be visualized, as marked by the dashed yellow lines. In pseudocolor PA images both along the long and the short axes of the entheses, strong signals reflecting the hyperemia can be clearly recognized. The enthesis inflammation detected by PA imaging was confirmed by the Doppler US imaging, which also showed active Doppler signals both along and across the entheses, although the Doppler signals along the entheses were not as strong.

Figure 3 shows the representative imaging results from a healthy volunteer. Similar to Fig. 2, the enthesis areas can be marked in B-mode US images. However, neither along the long axis of the enthesis nor across the enthesis can the PA images detect any obvious hyperemia signals, 
(a)

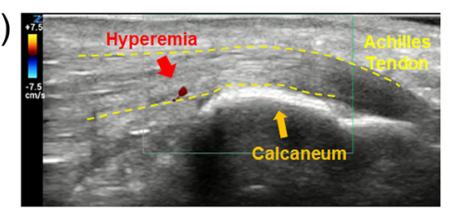

(c)

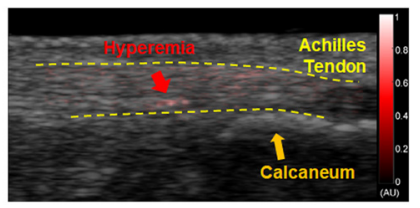

(b)

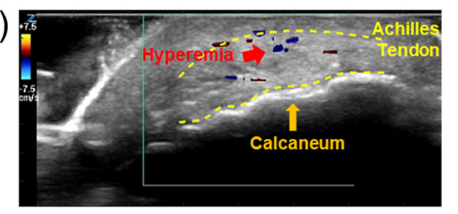

(d)

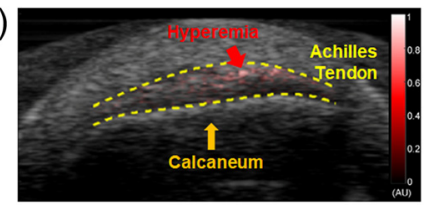

Fig. 2 US Doppler and PA imaging of an Achilles tendon (margins outlined by dotted line) of an SpA patient. (a) and (b) US Doppler images of the patient's Achilles tendon when scanned along the long and the short axes of the tendon, respectively. (c) and (d) PA images of the patient's Achilles tendon when scanned along the long and the short axes of the tendon, respectively. The pseudocolor PA images were superimposed on the gray-scale B-mode US images.

(a)

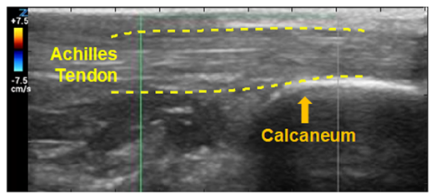

(c)

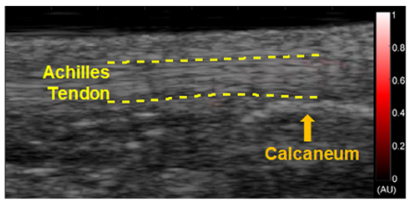

(b)

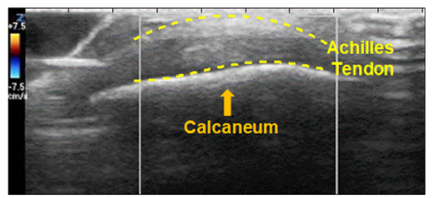

(d)

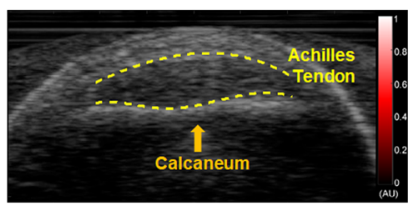

Fig. 3 US Doppler and PA imaging of an Achilles tendon of a normal volunteer. (a) and (b) US Doppler images of the volunteer's Achilles tendon when scanned along the long and the short axes of the tendon, respectively. (c) and (d) PA images of the volunteer's Achilles tendon when scanned along the long and the short axes of the tendon, respectively. No hyperemia noted.

indicating there was no enthesis inflammation. This finding was confirmed by the Doppler US imaging, which did not show any activation either along or across the enthesis.

To evaluate the performance of PA imaging for detecting enthesis inflammation, the results from $10 \mathrm{SpA}$ patients and 10 healthy volunteers were statistically compared. With the PA images acquired from each subject, two parameters that objectively reflect the severity of hyperemia were quantitatively analyzed, following the methods in previous publications. ${ }^{11,12}$ One parameter calculates the average intensity of all the colored pixels within the enthesis area in the PA image; the other parameter calculates the density of the colored pixels in the enthesis area, which is the ratio between the number of colored pixels and the number of all pixels of the tendon area. These two quantified PA parameters from SpA patients and healthy volunteers are compared in Figs. 4 and 5, where the results acquired along the entheses and across the entheses are studied individually. Figure 4(a) shows the box plots of the average intensities of colored pixels in the PA images along the entheses from the two groups, where the mean from the patient group is 4.53 times stronger than that from the healthy volunteer group. Figure 4(b) shows the box plots of the density of colored pixels in the PA images along the entheses from the two groups, where the mean from the patient is 11 times stronger than that from the healthy volunteer group. PA imaging results across the entheses, as shown in Figs. 5(a) and 5(b), demonstrate similar findings. The quantified average intensities of the colored pixels and the densities of the colored pixels from the SpA patient group have 7.5 fold and 13.75 fold enhancements, respectively, compared to the healthy volunteer group. To examine statistically significant differences between the two groups for each of the quantified parameters, a two tailed $t$-test was conducted using the built-in functions of MATLAB ${ }^{\circledR}$ (R2018b, Mathworks, Natick, Massachusetts). The calculated p values were $3.13 \times 10^{-6}$ and $5.29 \times 10^{-6}$, respectively, for the results of PA images along with the 

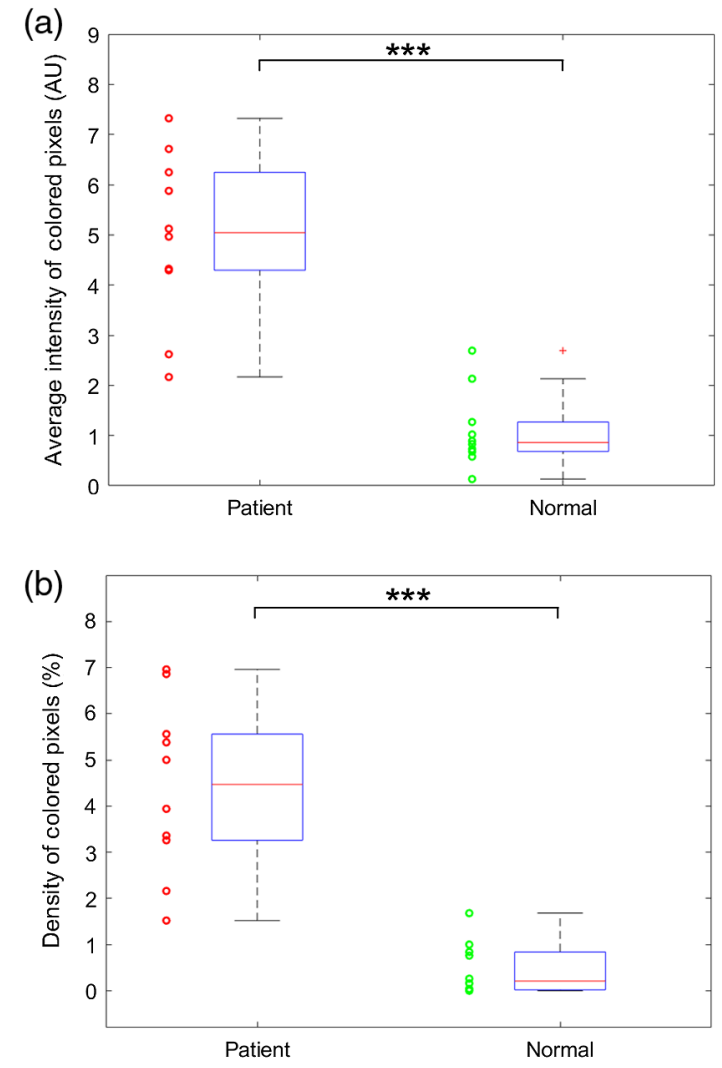

Fig. 4 Statistical analyses of the quantified PA imaging results reflecting the detected hyperemia along with the entheses. The results from the group of $10 \mathrm{SpA}$ patients are compared to the results from the group of 10 normal volunteers. (a) The average intensities of the colored pixels in the pseudocolor PA images scanned along the tendons from the two groups. (b) The density of the colored pixels in pseudocolor PA images scanned along the tendons from the two groups. ${ }^{* \star *}$ indicates $p<0.001$.

entheses in Figs. 4(a) and 4(b). The $p$ values from PA imaging results across the entheses were $1.66 \times 10^{-3}$ and $4.26 \times 10^{-3}$ in Figs. 5(a) and 5(b). The statistical results of the $t$-tests indicate that each quantified parameter can successfully differentiate the SpA patient group and the healthy volunteer group.

Statistical analysis was also conducted to compare the Doppler US images from the $10 \mathrm{SpA}$ patients and the 10 healthy volunteers. The results in Fig. 6 quantified the percentages of the colored pixels that present Doppler signals in the tendon area. The $t$-test comparing the Doppler US images from the two subject groups led to a $p$ value of $1.55 \times 10^{-6}$.

\section{Conclusion and Discussion}

Enthesitis is part of the ASAS criteria for SpA and can help distinguish this distinct pathological entity from RA; ${ }^{19,20}$ in addition, it could be an important biomarker in assessing treatment response or disease remission. Past modalities for evaluation of the presence/absence and severity of enthesitis include, but are not limited to, clinical exam, conventional radiography, US, and MRI. Each of these techniques has pros and cons associated with them. For example, clinical assessment of enthesitis has been organized as outcome measures (Maastricht Ankylosing Spondylitis Enthesis Score, Spondyloarthritis Research Consortium of Canada, and Leeds Enthesitis Index) but have never been validated to be used in SpA and have limitations in term of reliability, validity, and sensitivity. ${ }^{21}$ In addition, many of the entheseal points are around areas of accepted tender points for fibromyalgia (prevalent in $\mathrm{SpA}$ ), which leads to the possibility of misclassification. Conventional radiography detects later stage sequelae of enthesitis such as large erosions, but is not able to detect or quantify smaller erosions or earl soft tissue changes. 

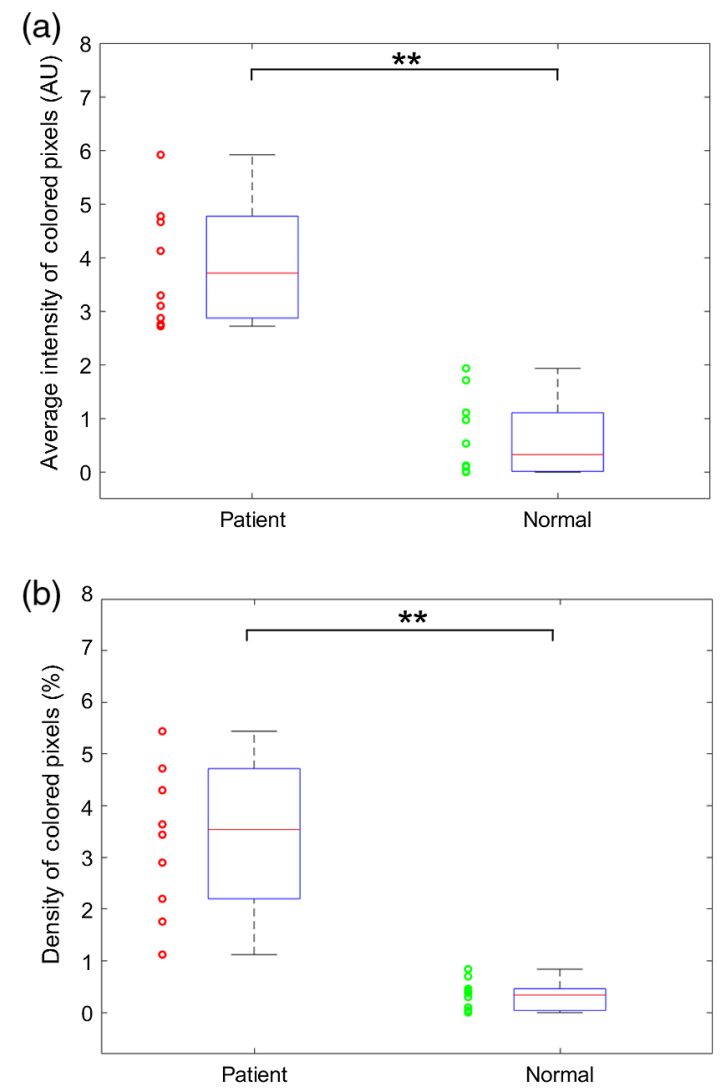

Fig. 5 Statistical analyses of the quantified PA imaging results reflecting the detected hyperemia across with the entheses. The results from the group of $10 \mathrm{SpA}$ patients are compared to the results from the group of 10 normal volunteers. (a) The average intensities of the colored pixels in the pseudocolor PA images scanned acrossed the tendons from the two groups. (b) The density of the colored pixels in pseudocolor PA images scanned acrossed the tendons from the two groups. ${ }^{* *}$ indicates $p<0.005$.

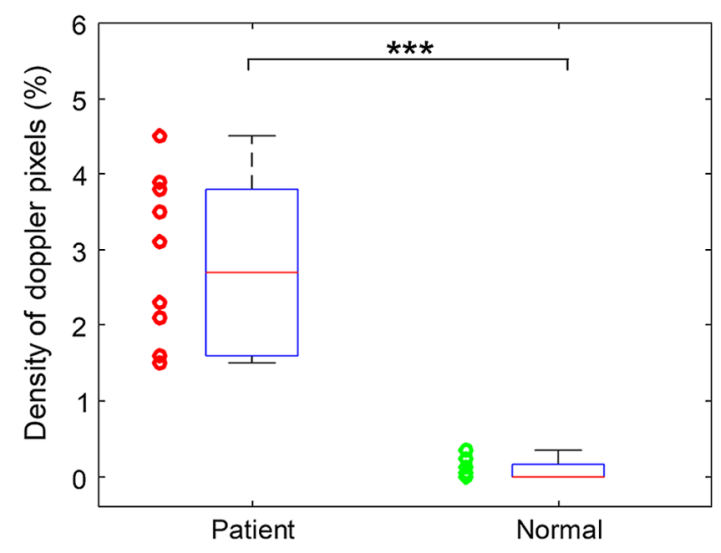

Fig. 6 Density of the colored pixels in the US Doppler images scanned along the tendons from the two groups including $10 \mathrm{SpA}$ patients and 10 normal volunteers, respectively. ${ }^{* \star *}$ indicates $p<0.001$.

MRI is a solid modality to evaluate enthesitis, but is expensive and not easily accessible. US is widely used because it is inexpensive, accessible, nonionizing, noninvasive, produces real-time images, and can document both bony abnormalities such as erosions along with soft tissue inflammatory characteristics. US, however, is highly operator dependent. ${ }^{22} \mathrm{PA}$ imaging is a 
potentially excellent imaging modality for enthesitis as it offers excellent sensitivity in assessing hyperemia and can also simultaneously take advantage of all of US's imaging capabilities.

In this work, via an initial clinical study on SpA patients and healthy volunteers, the emerging PA imaging technique's clinical potential for the detection of enthesis inflammation was explored. The PA images reflecting hyperemia were acquired using a PA and US combined system where the PA light source is based on two LED arrays. Due to its unique advantages over conventional laser-based PA imaging systems, such as portability, the LED-based system can be more beneficial as a point of care device for screening in a large population.

The results from this initial clinical study demonstrated that the PA images acquired at an 850-nm wavelength shows high sensitivity to enthesis inflammation and can easily detect the spatially distributed hyperemia both along the long axis and the short axis of the affected entheses. Despite the low energy of the light pulse $(400 \mu \mathrm{J})$ utilized, the imaging depth enabled by this LED PA imaging system is sufficient to cover the entire Achilles tendon or other superficial tendons. In the statistical results, PA imaging has demonstrated statistical significance to differentiate patients and healthy control subjects with low $p$ values $<0.005$. In a previous study, ${ }^{12}$ a comparison of PA and US Doppler for imaging of inflammatory arthritis was conducted, which demonstrated that, by leveraging the highly sensitive optical contrast, the PA imaging can achieve better sensitivity in detecting mild inflammation in human peripheral joints.

This clinical study has some limitations. The tendon insertion images acquired in these studies are all two-dimensional (2D). Although acquiring 2D images by the handheld probe is convenient and fits into the current clinical protocol, 2D images along arbitrary imaging planes make the imaging results highly dependent on the operators and difficult for quantitative assessment. Hence, our plan includes developing a three-dimensional (3D) imaging of tendon insertion inflammation, which could present volumetric information for a more accurate evaluation of hyperemia. Another limitation of the current LED technology is that the PA imaging system used in this study has limited optical wavelength options. As a result, all the images were acquired at a single wavelength of $850 \mathrm{~nm}$. Our plan includes PA imaging at two or more wavelengths, which could enable quantitative mapping of hypoxia (i.e., decreased blood oxygen saturation level) as another essential functional hallmark of tendon inflammation. This could be achieved by utilizing either dual-color LED arrays or other light sources working at multiple wavelengths. Despite these limitations, this initial clinical study has successfully demonstrated that PA imaging, as a safe and effective functional imaging modality, could offer an additional practical point of care tool for clinical diagnosis and treatment evaluation of SpA. This study extended the imaging ability for angiogenic microvessels to human tendon enthesitis. Encouraged by the promising results of the PA hyperemia imaging, we will move on to an advanced imaging system performing PA imaging together with US Doppler in a future study. Such a system that can obtain additional Doppler images simultaneously with PA imaging in tendon enthesitis will enable a more accurate comparison between the modalities.

\section{Disclosure}

The authors declare no conflict of interest.

\section{Acknowledgments}

This research was funded in part by the National Institutes of Health (Grants No. R01AR060350 and No. R37CA22282902).

\section{References}

1. C. G. Helmick et al., "Prevalence of psoriasis among adults in the U.S.: 2003-2006 and 2009-2010 National Health and Nutrition Examination Surveys," Am. J. Prevt. Med. 47(1), 37-45 (2014).

2. J. Brockbank and D. Gladman, "Diagnosis and management of psoriatic arthritis," Drugs 62(17), 2447-2457 (2002). 
3. J. Sieper et al., "The Assessment of Spondylo Arthritis international Society (ASAS) handbook: a guide to assess spondyloarthritis," Ann. Rheum. Dis. 68(Suppl), iil-44 (2009).

4. E. Feldtkeller et al., "Age at disease onset and diagnosis delay in HLA-B27 negative vs. positive patients with ankylosing spondylitis," Rheumatol. Int. 23(2), 61-66 (2003).

5. V. Wright and J. M. Moll, "Psoriatic arthritis," Bull. Rheum. Dis. 21(5), 627-632 (1971).

6. X. Wang et al., "Imaging of joints with laser-based photoacoustic tomography: an animal study," Med. Phys. 33(8), 2691-2697 (2006).

7. J. R. Rajian et al., "Characterization and treatment monitoring of inflammatory arthritis by photoacoustic imaging: a study on adjuvant-induced arthritis rat model," Biomed. Opt. Express 4(6), 900-908 (2013).

8. J. R. Rajian, G. Girish, and X. Wang, "Photoacoustic tomography to identify inflammatory arthritis," J. Biomed. Opt. 17(9), 096013 (2012).

9. X. Wang, D. L. Chamberland, and D. A. Jamadar, "Noninvasive photoacoustic tomography of human peripheral joints toward diagnosis of inflammatory arthritis," Opt. Lett. 32(20), 3002-3004 (2007).

10. G. Xu et al., "Photoacoustic and ultrasound dual-modality imaging of human peripheral joints," J. Biomed. Opt. 18(1), 010502 (2013).

11. J. Jo et al., "A functional study of human inflammatory arthritis using photoacoustic imaging," Sci. Rep. 7(1), 15026 (2017).

12. J. Jo et al., "Detecting joint inflammation by an LED-based photoacoustic imaging system: a feasibility study," J. Biomed. Opt. 23(11), 110501 (2018).

13. Y. Zhu et al., "Light emitting diodes based photoacoustic imaging and potential clinical applications," Sci. Rep. 8(1), 9885 (2018).

14. C. P. Favazza, L. A. Cornelius, and L. V. Wang, "In vivo functional photoacoustic microscopy of cutaneous microvasculature in human skin," J. Biomed. Opt. 16(2), 026004 (2011).

15. A. Hariri et al., "The characterization of an economic and portable LED-based photoacoustic imaging system to facilitate molecular imaging," Photoacoustics 9, 10-20 (2018).

16. K. Joseph Francis et al., "Tomographic imaging with an ultrasound and LED-based photoacoustic system,” Biomed. Opt. Express 11(4), 2152-2165 (2020).

17. E. Qvistgaard et al., "Quantitative ultrasonography in rheumatoid arthritis: evaluation of inflammation by Doppler technique," Ann. Rheum. Dis. 60(7), 690-693 (2001).

18. L. Terslev et al., "Doppler ultrasound and magnetic resonance imaging of synovial inflammation of the hand in rheumatoid arthritis: a comparative study," Arthritis Rheum. 48(9), 2434-2441 (2003).

19. J. F. Merola, L. R. Espinoza, and R. Fleischmann, "Distinguishing rheumatoid arthritis from psoriatic arthritis," RMD Open 4(2), e000656 (2018).

20. J. Belasco and N. Wei, "Psoriatic arthritis: what is happening at the joint?" Rheumatol. Ther. 6(3), 305-315 (2019).

21. P. J. Mease, "Measures of psoriatic arthritis: tender and swollen joint assessment, Psoriasis Area and Severity Index (PASI), Nail Psoriasis Severity Index (NAPSI), Modified Nail Psoriasis Severity Index (mNAPSI), Mander/Newcastle Enthesitis Index (MEI), Leeds Enthesitis Index (LEI), Spondyloarthritis Research Consortium of Canada (SPARCC), Maastricht Ankylosing Spondylitis Enthesis Score (MASES), Leeds Dactylitis Index (LDI), Patient Global for Psoriatic Arthritis, Dermatology Life Quality Index (DLQI), Psoriatic Arthritis Quality of Life (PsAQOL), Functional Assessment of Chronic Illness TherapyFatigue (FACIT-F), Psoriatic Arthritis Response Criteria (PsARC), Psoriatic Arthritis Joint Activity Index (PsAJAI), Disease Activity in Psoriatic Arthritis (DAPSA), and Composite Psoriatic Disease Activity Index (CPDAI)," Arthritis Care Res. (Hoboken) 63(Suppl. 11), S64-S85 (2011).

22. C. Bakewell et al., "Imaging techniques: options for the diagnosis and monitoring of treatment of enthesitis in psoriatic arthritis," J. Rheumatol. 47(7), 973-982 (2020).

Janggun Jo received his $\mathrm{PhD}$ in bioengineering from the University of Kansas. He finished a postdoctoral fellowship at the University of Michigan. His research interests include photoacoustic imaging modality including optical and ultrasound imaging as well as high intensity focused ultrasound treatment. 
Guan Xu is an assistant professor with the Department of Ophthalmology and Visual Sciences and Biomedical Engineering at the University of Michigan. He received his BS and $\mathrm{PhD}$ degrees in electrical engineering from Hebei University of Technology in China and Oklahoma State University in the US in 2006 and 2011, respectively.

Elena Schiopu is a board-certified rheumatologist and has been involved for 12 years in providing care for a variety of patients with systemic inflammatory illnesses. Her research interests included the connection between systemic sclerosis and atherosclerosis and she has studied the long-term survival of patients with idiopathic inflammatory myopathies (IIM). While gathering experience in the details of controlled clinical outcomes research, she has noted the significant need to standardize RA outcomes assessments and to optimize objective disease activity status.

David Chamberland received his BS degree in electrical engineering from the University of Portland (Oregon) and his MD degree from Oregon Health Sciences University. He completed his internal medicine residency and rheumatology fellowship at the University of Utah and University of Michigan, respectively. He is board certified both in internal medicine and rheumatology. He currently has an appointment in the Division of Rheumatology at the University of Michigan as a research investigator. His research interests include photoacoustic applications in medicine including inflammatory arthritis.

Girish Gandikota is a professor of radiology at the University of Michigan specializing in Musculoskeletal Radiology. He received his MBBS from Stanley Medical College, Chennai, India, and did his postgraduate training in the UK. He obtained board certifications in surgery (FRCS) and radiology (FRCR) in the UK. He had the opportunity to do a fellowship in Musculoskeletal Radiology at the McMaster University, Hamilton, Canada. He has received American Board of Radiology (ABR) certification. He also obtained ultrasound Registered in Musculoskeletal (RMSK) certification from American Registry for Diagnostic Medical Sonography (ARDMS). He is also the director of Domino Farms radiology. He is the vice-chair of the Assessment Oversight Team (AOT) RMSK-ARDMS appointed for musculoskeletal physician examination.

Xueding Wang is currently a professor at the Department of Biomedical Engineering, University of Michigan, holding an adjunct associate professor position in the Department of Radiology. He has extensive experience in imaging system development and adaptation of diagnostic technology to research and clinical managements. Sponsored by NIH, NSF, DoD, and other funding agencies, his research has led to over $100+$ peer-reviewed publications. He was the recipient of the Sontag Foundation Fellow of the Arthritis National Research Foundation in 2005, Joint Research Fund for Overseas Chinese Scholars and Scholars in Hong Kong and Macao from National Science Foundation of China in 2011, and the Distinguished Investigator Award of the Academy of Radiology Research in 2013. 